\title{
Una década enseñando e investigando en Educación Alimentaria para Maestros
}

\section{A decade teaching and researching in Food Education for Teachers}

\author{
Maximiliano RODRIGO VEGA, José Manuel EJEDA MANZANERA y Manuela \\ CABALLERO ARMENTA \\ Universidad Complutense de Madrid
}

Recibido: Septiembre 2012

Aceptado: Diciembre 2012

\section{Resumen}

La mejora de la Educación en Alimentación en colectivos como Maestros constituye un campo de interés dada su importancia estratégica de cara a ayudar a prevenir desde la escuela los altos índices de obesidad que se están dando actualmente. De hecho desde el curso 1997-98 colaboramos en la introducción de una nueva materia de estudio en la Facultad de Educación de la UCM de Madrid relacionada con esta temática. A la par hemos ido realizando distintas investigaciones tanto en la evolución de conocimientos como en la descripción de comportamientos de hábitos alimentarios de distintos grupos de futuros profesores con los que hemos trabajado. Dado que ha trascurrido más de una década desde que empezó toda esta labor educadora e investigadora nos ha parecido de interés realizar una síntesis actualizada que refleje en qué situación se encuentra el diseño didáctico sobre el que actualmente abordamos la Educación Alimentaria para Maestros y cuáles son los principales datos investigadores que actúan como condicionantes en nuestra labor, así como los principales retos futuros que tenemos.

Palabras clave: Educación para la salud, hábitos saludables, formación del profesorado, educación en alimentación.

\begin{abstract}
The improvement of food education in a Group of teachers contributes to a field of interest due to the strategic importance of preventing obesity now prevalent. In fact since the academic year 1997 /1998 we have been collaborating by introducing a new lecture in the University of Education from the UCM in Madrid, which is related to this topic. At the same time, we have been carrying out research on both, the development of knowledge and the description of food behaviour habits from different groups of future teachers that we have worked with. Due to the fact that this research took place more than a decade ago since having started all this research and educational action, we have deemed it necessary to engage in updated research, which reflects what situation the didactic design faced nowadays in food education is actually in for


teachers, and which are the main research data items that perform as keys for our research, thus the main future challenges that we have.

Keywords: Education for health, healthy habits, teacher training, nutrition education.

\section{La Educación Alimentaria como temática clave de Educación para la Salud (EpS) en la Formación de los futuros Profesores}

La enseñanza y promoción de la Alimentación saludable consideramos debería ser un objetivo preferente en la formación de los futuros profesores y educadores. Esta premisa ha perseguido nuestros esfuerzos formadores e investigadores durante más de una década en un campo tan clave como es el de la Formación de Maestros. Aquí vamos a ir presentando las directrices actualizadas que guían nuestra docencia en torno a una materia sobre "Educación Alimentaria para Maestros" y algunos de los datos más representativos de las investigaciones que hemos llevado a cabo a la par que nuestra docencia en torno a ella.

Podemos definir a la Educación Alimentaria como el conjunto de métodos y estrategias que ayudan a que el individuo y la población en general pueda llevar a cabo una Alimentación saludable.

En esencia la Educación Alimentaria y nutricional pretende que se adopten comportamientos que mejoren la salud a través de una serie de experiencias de aprendizaje, modificando estos comportamientos y los determinantes que actúan sobre ellos (Martínez et al., 2007, p.140).

Hoy en día se están dando altos índices de obesidad entre la población empezando a ser éste un problema de salud pública que es necesario reducir. Así, la Educación Alimentaria está emergiendo como una herramienta imprescindible para paliar esta problemática. En este sentido organismos como la Organización Mundial de la Salud (OMS, 1998, 2002 y 2006; WHO, 1986) están liderando múltiples iniciativas que buscan mejorar los hábitos alimentarios empezando por la población escolar.

En la dirección indicada, las instituciones sanitarias del Estado español y sus comunidades autónomas se han interesado por buscar en los centros educativos, y especialmente en el profesorado, un soporte importante para impulsar sus actuaciones en temas de alimentación, de salud y de consumo responsable (véanse, por ejemplo, los desarrollos normativos sobre los distintos currículos: BOE 2006 a, b y c). Igualmente, hace poco en España se promulgó la LEY de SEGURIDAD ALIMENTARIA Y NUTRICIÓN (Ley 17/2011) en el BOE nº 160 de 6 de Julio de 2011. En esta norma especial interés tiene el Capítulo VII dirigido entre otros aspectos a mejorar la Educación Alimentaria y dentro de él destacamos el siguiente Artículo 40.1 que dice literalmente:

Las autoridades educativas competentes promoverán la enseñanza de la nutrición y alimentación en las escuelas infantiles y centros escolares,... A tal efecto, se introducirán contenidos orientados a la prevención y a la concienciación sobre los 
beneficios de una nutrición equilibrada en los planes formativos del profesorado (p. 71305).

En la misma línea de integrar en los programas de Formación Inicial del Profesorado de enseñanza obligatoria aspectos de EpS, se planteó el diseño de una asignatura titulada "La Alimentación Humana para Maestros" (Rodrigo, 1999 y 2000) que ha sido el origen de lo que aquí presentaremos actualizado y renovado.

El nacimiento de dicha asignatura guarda relación con nuestra preocupación por introducir aspectos de EpS en la formación de futuros Maestros. En el año 1995 abordamos una investigación sobre las preferencias que nuestros alumnos (participaron 318 futuros Maestros) tenían sobre distintas posibilidades de temáticas de EpS en los nuevos Planes de estudio que entonces se abordaban. Nuestra sorpresa coincidió con nuestro interés: La Educación Alimentaria fue la temática más demandada por la gran mayoría de los alumnos (Rodrigo, 1995). Esto nos llenó de satisfacción y de energía para emprender actividades de enseñanza sobre ella. Además, en la Alimentación se da la importancia de ser una actividad básica del ser humano, de tener un cuerpo de conocimientos amplio pero que se puede matizar y adaptar a nuestros intereses educativos y la actividad alimentaria tiene un amplio componente educativo desde su origen. Consideramos que en ella confluye lo básico de cualquier temática de EpS y es ejemplarizante para profundizar en el enseñar el cómo abordar la EpS en la escuela obligatoria. Seguidamente presentaremos primero nuestra propuesta docente que se ha ido conformando a lo largo de estos años y posteriormente distintas investigaciones que de una manera $\mathrm{u}$ otra también han determinado y condicionan nuestra apuesta didáctica.

\section{Descripción de la Asignatura base sobre la que hemos enseñado e investigado}

\section{Contexto y Objetivos}

El diseño de la asignatura que aquí presentamos, ha ido evolucionando y seguirá seguramente evolucionando (es mejorable), recordemos que el antecedente fue una optativa de 4,5 créditos que hemos impartido durante más de diez años denominada "Alimentación Humana en la Escuela" y tenemos la satisfacción de haber podido dar clase en esta materia a varios centenares de alumnos.

La asignatura anterior se ha venido impartiendo hasta el curso 2011-12 en el segundo año de Magisterio, a estudiantes de 21 a 25 años de edad, de la Facultad de Educación de la UCM (véase su diseño inicial en Rodrigo, 1999 y 2000). A partir del curso 2012-13 la nueva asignatura, que en parte coge el testigo de las anteriores, se denomina "Fundamentos y didáctica de la Alimentación", se integra como Obligatoria, de 6 créditos, para los Maestros de Educación Infantil en el $4^{\circ}$ año $\left(7^{\circ}\right.$ semestre) de su formación en la UCM (ver BOE ${ }^{\circ} 157$ de 29-06-11, Resolución de la UCM de 7-0610). No entramos a valorar la necesidad, para nosotros evidente, de que también se debería ofrecer a futuros Maestros de Primaria. 
Igualmente deseamos manifestar que a partir del hecho de que el legislador quiso introducir una Materia de Alimentación en los diseños de los Planes de estudio de Graduados en Magisterio como obligatoria-troncal al menos en Maestros de Ed. Infantil (BOE $\mathrm{n}^{\circ} 132$ de 29-12-07; Orden Ministerial ECI /3854/ de 27-12-2007) consideramos que sería interesante una investigación sobre cómo se están abordando los diseños de los planes docentes de esta Materia que han ido apareciendo publicados en el BOE por parte de las distintas universidades españolas. Por lo que hemos ido constatando, a nuestro modo de entender, la realidad docente de esta Materia podría estar desvirtuada en muchos de ellos. Esto queda para ser abordado en otra ocasión, animo a posibles estudiosos del tema a indagarlo, mientras tanto nosotros intentaremos en las siguientes páginas ofrecer las principales directrices de nuestra docencia.

Los objetivos generales de la asignatura actual de Alimentación para Maestros son:

a) Aprendizaje de los principales conceptos relacionados con la temática. Potenciar en nuestros alumnos un espíritu crítico en el análisis de cualquier contenido informativo relacionado con las cuestiones básicas de la Alimentación.

b) La revisión crítica de las propias pautas de alimentación, como paso previo para posibilitar cambios en sus creencias, actitudes y hábitos alimentarios.

c) Ofrecer experiencias educativas que guarden relación con el tema y que puedan servir de referencia para su futura labor docente.

Otros objetivos, derivados de los anteriores serían:

- Clarificar la diferencia entre Alimentación y Nutrición.

- Entender la "práctica de la higiene-alimentaria y la práctica de la elección de alimentos saludables" como las cuestiones básicas de la Educación Alimentaria a nivel escolar.

- Identificar los principales nutrientes de los Alimentos más comunes.

- Relacionar los nutrientes con su función dietética.

- Analizar y comprender el sentido didáctico de las Guías Alimentarias para la práctica de una dieta equilibrada.

- Revisión crítica de las propias pautas de alimentación. Presentar un trabajo individual de análisis y revisión (cuaderno personal evaluable).

- Intentar proponer- modificar algunos hábitos de Alimentación y de higiene.

- Identificar y analizar factores sociológicos, económicos y educativos en las formas de Alimentación.

- Conocer algunos aspectos de la diversidad cultural en Alimentación.

- Fomentar el espíritu de solidaridad con los que menos tienen. 
- Expresar sus propias opiniones, escuchar a los demás; favorecer el trabajo en grupo.

- Favorecer la formación del futuro Profesor analizando ejemplos de diseños didácticos y de recursos educativos en Educación Alimentaria.

Tanto en la planificación como en el desarrollo de la propuesta se ha seguido un modelo de enseñanza-aprendizaje que permita hacer que los estudiantes sean conscientes de su conocimiento previo, de sus creencias y de sus hábitos, de las coincidencias y discrepancias con sus puntos de vista y los de sus compañeros; facilitar el aprendizaje de nuevo conocimiento; en definitiva mejorar su saber y sus actitudes ante la problemática de la Alimentación y en última instancia revisar sus hábitos aprendiendo a practicar una Alimentación saludable. A partir de aquí sentar las bases para poder enseñar a otros. Seguidamente pasamos a describir la organización docente.

\section{Diseño general}

Para el diseño de las temáticas de la "Alimentación- Nutrición" como parte de la EpS, Gavidia et al., (1993), establecen cuatro fuentes en el diseño del currículo que a nosotros nos han servido de guía y hemos matizado para nuestros intereses en la propuesta de la Asignatura de Alimentación para Maestros que impartimos y son:

- Desde la fuente Sociológica, que se basaría en las nuevas necesidades sociales y las demandas que hace la sociedad para que ciertos temas se desarrollen en la escuela, por considerarlos necesarios para los ciudadanos del futuro. Sin duda nuestro interés prioritario en este campo se centra en dos fines: "Que los ciudadanos aprendan a saber elegir alimentos y que en su vida sean solidarios"

- Desde la fuente Epistemológica, se fundamentaría en la estructura interna de la disciplina a desarrollar, los conocimientos científicos, su historia y su estado actual. Establece las relaciones interdisciplinares pertinentes, diferencia lo fundamental de lo accesorio y presenta una metodología de trabajo. De aqui se derivan dos fines en nuestro diseño: "Entender el significado de las Guías Alimentarias como instrumentos de ordenamiento de la alimentación equilibrada" y de suma importancia para la didáctica de la dieta. Asimismo "aprender a ser críticos con las informaciones alimentarias que frecuentemente inundan nuestros medios de comunicación"

- Desde la fuente Psicológica, que se nutre del conocimiento de los procesos de desarrollo intelectual, procesual y psicomotor de los alumnos, las regularidades que presentan las diferentes etapas evolutivas, las diferentes etapas evolutivas, las diferencias individuales existentes, y los mecanismos y procesos de aprendizaje. Aquí cobra especial importancia el desarrollo de actitudes y pautas de comportamiento, que configuran un determinado estilo de vida. Nuestros alumnos ante todo son adultos y a ellos va dirigida en primer lugar la docencia (serán en un futuro la mayoría de ellos Profesores, pero ante todo son 
personas con un comportamiento alimentario concreto). Ello determina un fin primordial necesario que consiste en hacer efectivo el análisis concreto de su comportamiento alimentario, conocer, entender y buscar las bases de motivación para practicar una dieta saludable.

- Desde la fuente Pedagógica, que considera la intervención del profesorado en el hecho didáctico, diseñando y llevando a efecto una adecuada secuencia de Enseñanza-Aprendizaje (E-A) todo ello basado en la experiencia docente y en las investigaciones psicopedagógicas. En nuestro diseño, "el aprender a interpretar su propia dieta es la base didáctica sobre la que poder construir la didáctica de la dieta para otros", luego los matices didácticos irán apareciendo con cada situación concreta y se trabajan ejemplos en el aula. Así, también se busca que el alumnado conozca una serie de recursos actualizados en torno a la didáctica de la dieta adaptada a distintas etapas de la Educación Básica, especialmente para la Educación Infantil y Primaria. Nuestra apuesta es la formación de un Profesor que en un Centro se pueda enfrentar a diseños de Educación Alimentaria enfocables a distintos actores del mundo educativo desde los Padres a las Empresas del comedor escolar o los compañeros del Equipo docente.

El organigrama docente se compone de una conjunción de dos módulos: TeóricoPráctico que se estructuran según los principales conceptos que distintos autores asocian con el tema y que hemos ido adaptando a la realidad del aula. Estos módulos se encuentran distribuidos en el Programa como se muestran en los Cuadros 1 y 2 . En el Cuadro 1 se describen sintéticamente los principales tiempos y partes de la asignatura; igualmente, en la base del Cuadro 1 se observan los principales momentos evaluadores. Ya en el Cuadro 2 se presentan los dos módulos de diseño y su encaje e interrelación. Asimismo los principales conocimientos básicos que abordamos se encuentran relacionados como se muestran en la Figura 1. 


\begin{tabular}{|c|c|c|}
\hline $\begin{array}{l}\text { APARTADO } \\
\text { del Programa }\end{array}$ & $\begin{array}{l}\text { SIGNIFICADOS } \\
\text { Primordiales }\end{array}$ & $\begin{array}{l}\text { TEMPORALIDAD } \\
\text { (Semanas) }\end{array}$ \\
\hline Introducción al Curso & $\begin{array}{l}\text { Presentación general de las líneas de } \\
\text { Metodología de Trabajo a seguir. } \\
\text { Explicación de la evaluación*. } \\
\text { Análisis aproximado de la situación } \\
\text { Alimentaria de cada persona y del } \\
\text { grupo clase. }\end{array}$ & $1^{\mathrm{a}}$ y $2^{\mathrm{a}}$ \\
\hline $\begin{array}{l}\text { I- Significado Biológico de la } \\
\text { Alimentación-Nutrición }\end{array}$ & $\begin{array}{l}\text { Entender ¿cómo se conecta la } \\
\text { Alimentación y la Nutrición? }\end{array}$ & $3^{\mathrm{a}}$ y $4^{\mathrm{a}}$ \\
\hline $\begin{array}{l}\text { II- Fundamentos. ¿Qué tienen los } \\
\text { Alimentos? ¿Qué necesitamos? } \\
\text { ¿Cómo comer? } \\
\text { Dieta y etapas escolares. }\end{array}$ & $\begin{array}{l}\text { Trabajar-analizar-entender la dieta } \\
\text { personal. Conocer las cuestiones } \\
\text { básicas de las dietas en las etapas } \\
\text { escolares. }\end{array}$ & $5^{\mathrm{a}}$ a la $9^{\mathrm{a}}$ \\
\hline $\begin{array}{l}\text { III- Cuestiones sociales de interés } \\
\text { Educativo: } \\
\text { Preparación y conservación de } \\
\text { alimentos. } \\
\text { Educación del Consumidor. } \\
\text { Educación Alimentaria y } \\
\text { solidaridad. Salud y Alimentación }\end{array}$ & $\begin{array}{l}\text { Conocer y analizar distintas facetas } \\
\text { sociales que conectan la Alimentación } \\
\text { con el mundo Educativo. }\end{array}$ & $10^{\mathrm{a}}$ a la $12^{\mathrm{a}}$ \\
\hline $\begin{array}{l}\text { IV- Enfoques de Educación } \\
\text { Alimentaria }\end{array}$ & $\begin{array}{l}\text { Reflexiones y análisis en torno a } \\
\text { recursos y diseños educativos propios } \\
\text { del trabajo de la Educación } \\
\text { Alimentaria. }\end{array}$ & $12^{\mathrm{a}}$ a la $15^{\mathrm{a}}$ \\
\hline \multicolumn{3}{|c|}{$\begin{array}{l}\text { *EVALUACIÓN (Instrumentos): } \\
\text { - Cuestionario previo (evaluación inicial). } \\
\text { - Los alumnos deben elaborar además un dossier-cuaderno personal de su paso por la Asignatura en el } \\
\text { que van recogiendo todas sus actividades, se recoge para ser evaluado el día del Examen y se devuelve } \\
\text { posteriormente. Especial importancia tiene en el dossier el análisis y la valoración de su DIETA personal. } \\
\text { Esta actividad es NUCLEAR sin su desarrollo adecuado no se puede superar la asignatura. La } \\
\text { ponderación del DOSSIER en la nota final (siempre que como mínimo se dé la condición anterior), es del } \\
\mathbf{4 0} \% \text {. } \\
\text { - El seguimiento de las actividades de enseñanza y su entrega correspondiente en tiempo y forma se } \\
\text { valorará el } \mathbf{2 0 \%} \text { de la nota final. } \\
\text { - Examen escrito, en el que se valorarán los conocimientos básicos y el juicio crítico de cada alumno } \\
\text { sobre cuestiones que previamente se han analizado o estudiado en el desarrollo docente. Su cómputo } \\
\text { representará el } \mathbf{4 0 \%} \text { de la nota final. }\end{array}$} \\
\hline
\end{tabular}

Cuadro 1: Principales Partes del Programa de la asignatura de Alimentación para Maestros, su significado y temporalidad en nuestra programación. Igualmente se establecen los principales instrumentos de Evaluación* que se emplean. 


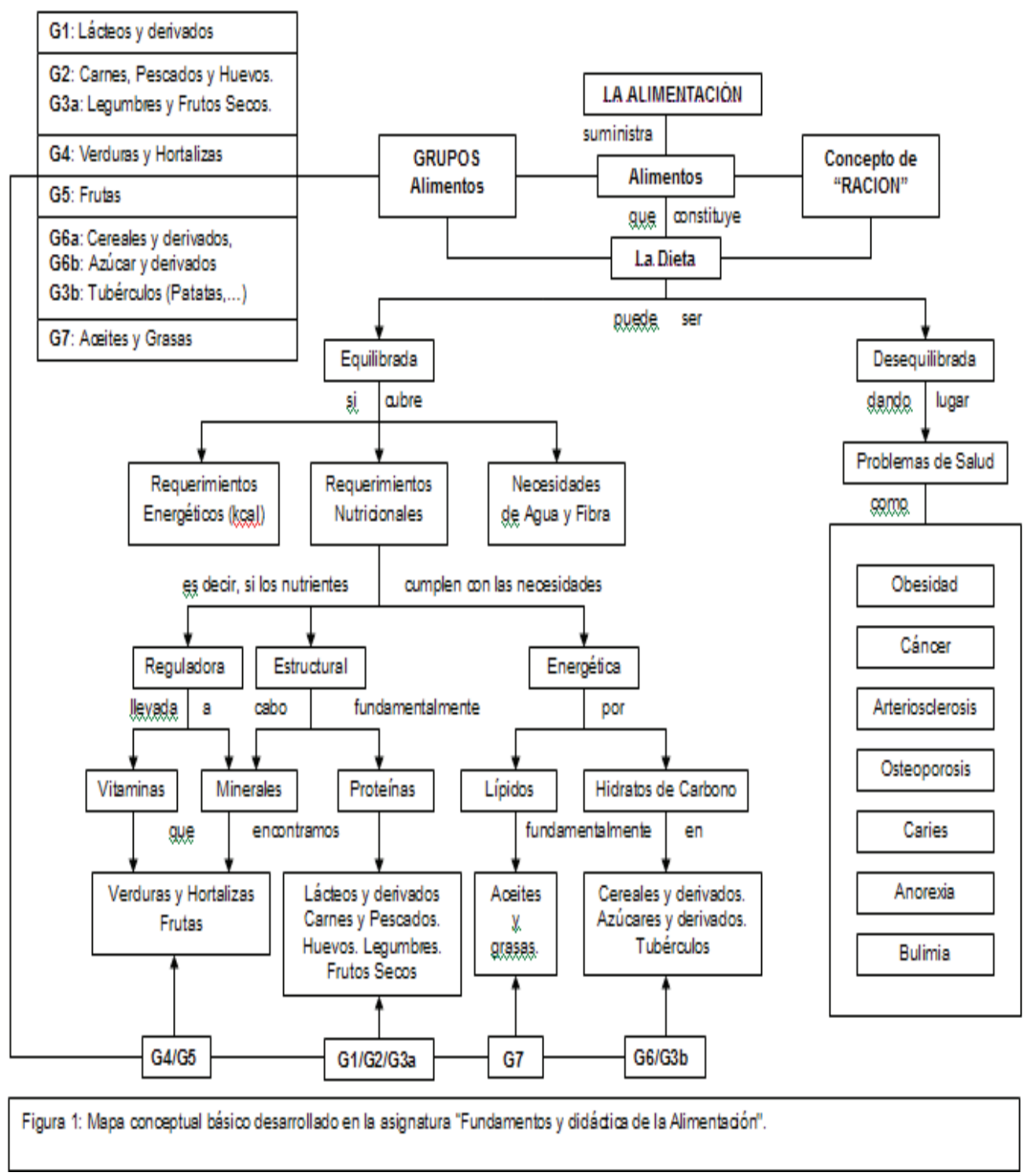




\begin{tabular}{|c|c|}
\hline $\begin{array}{c}\text { Módulo Teórico } \\
\text { (Temáticas clave) }\end{array}$ & $\begin{array}{l}\text { Módulo Práctico (ACTIVIDADES GUÍA*: } \\
\text { I, individual; Pg., en grupos; G, de clase). }\end{array}$ \\
\hline . Introducción.(Presentación-Motivación) & $\begin{array}{l}\text { ¿Cuál es nuestra situación?: } \\
\text { Act. (I): Cuestionario previo } \\
\text { Act. }(\mathrm{I} / \mathrm{G}): \text { Cálculo de ¿Pesos Ideales? }\end{array}$ \\
\hline - I. Significado biológico de la Alimentación. & $\begin{array}{l}\text { - I.: Sesión de vídeo sobre la conexión Alimentación- } \\
\text { Nutrición }(\mathrm{G}) \text {. }\end{array}$ \\
\hline $\begin{array}{l}\text { - II Fundamentos conceptuales. } \\
\text { - II.1.Principales nutrientes. } \\
\text { (Carbohidratos, Lípidos, Proteínas, Vitaminas, Agua - } \\
\text { Sustancias Minerales). }\end{array}$ & $\begin{array}{l}\text { - II.1. ¿Qué nutrientes fundamentales tiene un } \\
\text { alimento como la leche?: } \\
\text { Act. (Pg.): Experimental en laboratorio. }\end{array}$ \\
\hline $\begin{array}{l}\text { - II.2. Principales necesidades nutritivas humanas. } \\
\text { (Energéticas. Constructoras. Reguladoras). }\end{array}$ & $\begin{array}{l}\text { - II.2. ¿Cómo conseguir lo necesario?: } \\
\text { Act. (I,Pg.): Cálculo de Biotipos Energéticos. }\end{array}$ \\
\hline $\begin{array}{l}\text { - II.3. La dieta equilibrada. } \\
\text { (Grupos de alimentos. Raciones. Guías Alimentarias. } \\
\text { Dieta equilibrada). }\end{array}$ & $\begin{array}{l}\text { - II.3. ¿Que comes?: } \\
\text { Act. (I/G): registro semanal de dietas. } \\
\text { ¿Cómo mejorar la situación?: } \\
\text { Act. (I/G): Propuesta de mejoras. }\end{array}$ \\
\hline $\begin{array}{l}\text { - III Cuestiones sociales y culturales. (Sociología, } \\
\text { Etología y Ecología de la Alimentación. Higiene y } \\
\text { conservación de alimentos. Salud y dieta). }\end{array}$ & $\begin{array}{l}\text { - III. ¿Qué cuestiones sociales pueden interesarnos?: } \\
\text { Act. (Pg./G): Lectura y comentario de textos }\end{array}$ \\
\hline $\begin{array}{l}\text { - IV Cuestiones educativas. } \\
\text { ¿Planteamos una unidad didáctica? }\end{array}$ & $\begin{array}{l}\text {-IV ¿Cómo trabajar en el aula?: } \\
\text { Act. (Pg./G): Análisis de unidades didácticas. }\end{array}$ \\
\hline
\end{tabular}

Nota (*): Actividades Guía o tipo (I), (Pg.), (G): I-individual, Pg.- en grupos y G- de clase. Otras las definimos en función de la marcha del curso.

Cuadro 2: Módulos Teórico (Temáticas)-Práctico (Actividades Guía) de la asignatura de Alimentación para Maestros.

Los objetivos del Módulo Práctico (véanse los Cuadros 3 y 4) serían:

- Intentar conectar los conceptos desarrollados, con la realización de actividades prácticas individuales y grupales. Para tal fin cada alumno deberá tener un cuaderno de trabajo, de esta materia que irá complementando durante el curso y que será evaluable. Asimismo muchas actividades se recogen en el aula o se da un tiempo para la entrega y nos serán de utilidad para el seguimiento del alumnado.

- Resolver distintas situaciones-cuestiones planteadas que los acerquen a la problemática de la Alimentación desde distintas facetas: personales, sociales, desde la Salud o desde la Educación.

Este Módulo se ha estructurado en forma de preguntas sugerentes (o situaciones problemáticas) y actividades tipo que tienen como objetivo dar respuesta a tales cuestiones y de esta manera se va profundizando en los distintos aspectos teóricos tratados. Se basa en el planteamiento de cuestiones abiertas, que favorecen la implicación y el interés de los mismos estudiantes. Estas preguntas deben conectar con 
su bagaje de experiencias y conocimientos para que sean relevantes para ellos. Se diseñan para ayudar a los estudiantes a que sean capaces de entender cómo la Alimentación no sólo contempla aspectos biológicos, sino también culturales y sociales, ya que desarrollarán estrategias para fomentar su papel como consumidores alimentarios críticos o como futuros docentes sobre temáticas alimentarias.

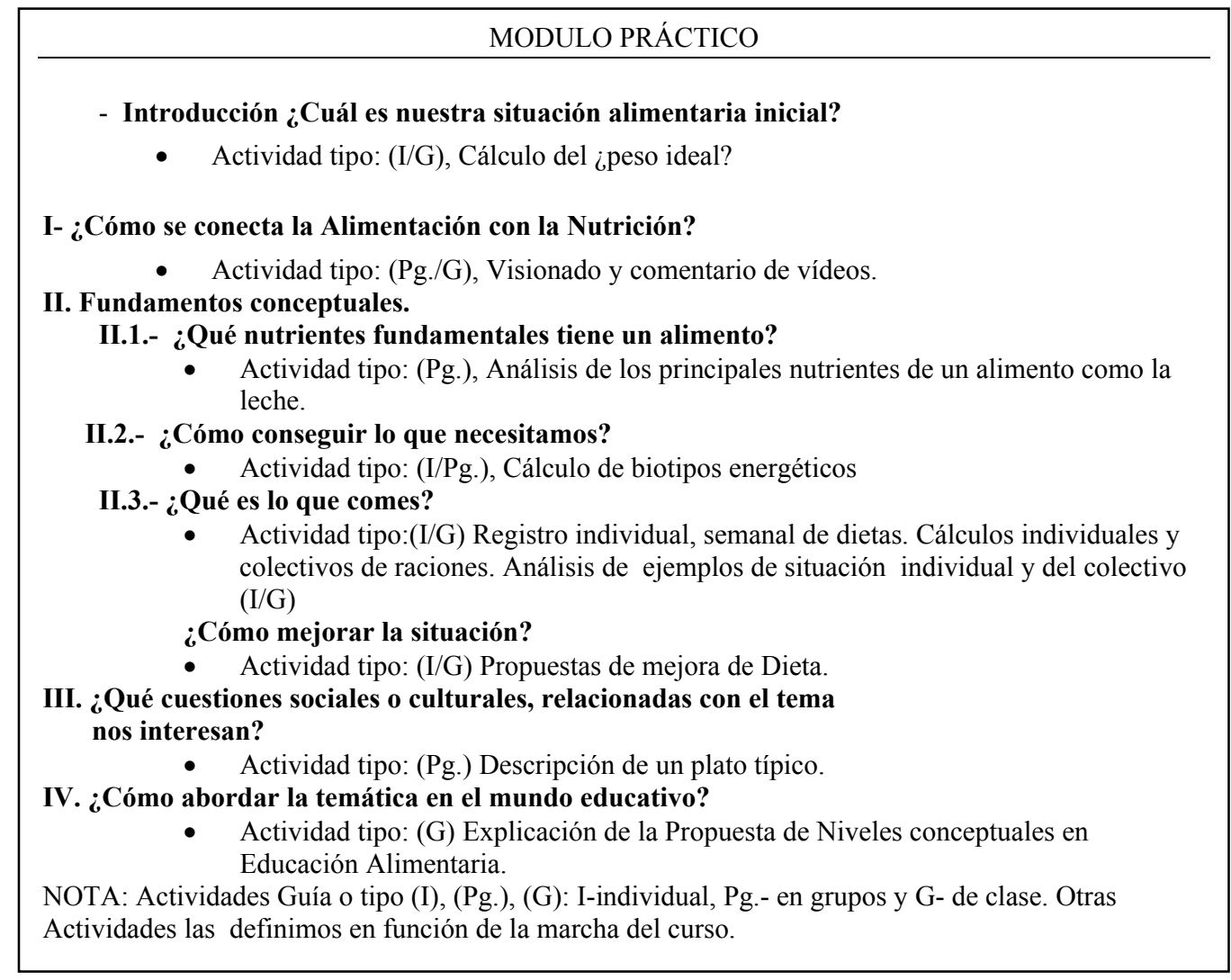

Cuadro 3: Estructura del Módulo Práctico de la asignatura de la Alimentación en torno a preguntas.

El Módulo Práctico es el que más suelen valorar los alumnos en sus comentarios de final de curso. Una de las claves del módulo es intentar partir de la concienciación del alumno sobre su situación, al parecer porque los acerca más a su realidad -véase Cuadro 3- (véase también Rodrigo, 1999 y 2000).

Por otro lado, para favorecer el seguimiento y evaluación de las actividades en él incluidas, es fundamental un cuaderno individual de trabajo que los alumnos deben entregar al finalizar el curso. En él no pueden faltar dos actividades nucleares: el análisis de su Peso/Talla (medida indirecta de su situación alimentaria) y el análisis 
sistemático de la dieta semanal que realizan, aspectos que son básicos en el desarrollo de nuestra propuesta didáctica.

La actividad del cálculo de parámetros como la relación Peso-Talla o el mal llamado Peso Ideal (¿?) por varios métodos tiene un claro objetivo de concienciar desde comienzo de curso a cada alumno de su situación alimentaria de una manera indirecta, pero reflexiva y crítica potenciando posturas que en Alimentación no deben ser rígidas. De esta manera, se pueden extraer comentarios de los cuadernos de trabajo como aparece descrito por Rodrigo (1999 y 2000). En ellos los alumnos aprenden a interpretar sus datos de Peso-Talla por varios métodos de análisis que a veces les llevan a resultados aparentemente contradictorios sobre su situación alimentaria.

Otra de las actividades clave consiste en el análisis sistemático (durante una semana) de la dieta real que realiza cada alumno para posteriormente revisar las situación individual en comparación con lo que podría ser más correcto. Para ello utilizamos el concepto de "ración alimentaria" adaptado a la actualidad y a nuestros alumnos (a partir de López Nomdedeu, 1974) y trabajamos profusamente el sentido didáctico de las "Guías Alimentarias" en especial utilizamos una reformulación de la Rueda de los Alimentos clásica española (véase lo indicado en Rodrigo y Ejeda, 2010). El objetivo es simplificar los cálculos para que los alumnos dispongan de un método sencillo y fácil de utilizar en su vida cotidiana. Esta actividad es clave para la revisión de hábitos diarios -reconocidos muchas veces de una manera sorpresiva como inadecuada-. Igualmente consideramos esta actividad básica para transferir la didáctica de la dieta a otros como hemos expuesto detenidamente en 2010 y recordaremos de nuevo en las conclusiones.

Por último, añadir que estos replanteamientos de conductas se complementan con el análisis de cuestiones sociales y educativas completándose así el ciclo educativo que reiteramos pretendemos que sea vivo y vaya adaptándose a las nuevas circunstancias. Precisamente parte de la adaptación viene motivada por las Investigaciones que hemos ido realizando a la vez que nuestra docencia y que seguidamente presentamos de una manera resumida, especial hincapié haremos en los datos y conclusiones que actualmente conducen nuestros intereses educativos. 


\begin{tabular}{|c|c|c|c|}
\hline $\begin{array}{c}\text { Situación } \\
\text { problemática }\end{array}$ & $\begin{array}{l}\text { Actividades } \\
\text { Guía }\end{array}$ & Objetivos & Contenidos \\
\hline $\begin{array}{l}\text { ¿Cuál es nuestra } \\
\text { situación? }\end{array}$ & $\begin{array}{l}\text { Cálculo de peso } \\
\text { ¿ ideal? }\end{array}$ & $\begin{array}{l}\text { Motivación. Concienciar al } \\
\text { alumno de su propia situación } \\
\text { nutricional al analizar su } \\
\text { situación personal con respecto } \\
\text { al mal llamado peso ideal. }\end{array}$ & $\begin{array}{c}\text { Métodos para el } \\
\text { cálculo del peso/talla, } \\
\text { como aproximación a } \\
\text { la situación } \\
\text { alimentaria. }\end{array}$ \\
\hline $\begin{array}{c}\text { ¿Cómo conseguir } \\
\text { lo que } \\
\text { necesitamos? }\end{array}$ & $\begin{array}{l}\text { Análisis de los } \\
\text { principales nutrientes } \\
\text { de un alimento } \\
\text { Cálculo de los } \\
\text { biotipos }\end{array}$ & $\begin{array}{l}\text { Conocer las relaciones entre los } \\
\text { alimentos y los nutrientes. }\end{array}$ & $\begin{array}{c}\text { Los diferentes } \\
\text { nutrientes, } \\
\text { (carbohidratos, } \\
\text { proteínas, lípidos, } \\
\text { vitaminas, minerales y } \\
\text { agua). Tablas Guía }\end{array}$ \\
\hline $\begin{array}{l}\text { ¿Qué es lo que } \\
\text { comes? }\end{array}$ & $\begin{array}{l}\text { Registro individual } \\
\text { semanal. } \\
\text { Cálculos individuales } \\
\text { y colectivos de } \\
\text { raciones. Análisis de } \\
\text { situación. }\end{array}$ & $\begin{array}{l}\text { Explicitar ideas. Revisión } \\
\text { crítica de las propias pautas de } \\
\text { alimentación. Orientación } \\
\text { alimentaria. }\end{array}$ & \multirow{2}{*}{$\begin{array}{l}\text { Clasificación de los } \\
\text { alimentos } \\
\text { Concepto de "ración } \\
\text { alimentaria". } \\
\text { Guías Alimentarias } \\
\text { Recursos didácticos } \\
\text { Dieta equilibrada } \\
\text { Dieta en niños }\end{array}$} \\
\hline $\begin{array}{l}\text { ¿Cómo mejorar la } \\
\text { situación? }\end{array}$ & $\begin{array}{l}\text { Revisión de la Dieta. } \\
\text { Educación } \\
\text { Alimentaria escolar }\end{array}$ & $\begin{array}{c}\text { Detectar problemas dietéticos, } \\
\text { (conflicto cognitivo). Adoptar } \\
\text { nuevos hábitos relacionados a la } \\
\text { salud. }\end{array}$ & \\
\hline $\begin{array}{l}\text { ¿Qué cuestiones } \\
\text { sociales o } \\
\text { culturales, } \\
\text { relacionadas con } \\
\text { el tema nos } \\
\text { interesan? }\end{array}$ & $\begin{array}{c}\text { Lecturas y } \\
\text { comentarios de textos: } \\
\text { Análisis de etiquetas. } \\
\text { Preparación y } \\
\text { conservación de los } \\
\text { alimentos. Dieta } \\
\text { mediterránea } \\
\text { Anorexia y bulimia... }\end{array}$ & $\begin{array}{l}\text { Identificar y analizar factores } \\
\text { sociológicos, económicos y } \\
\text { educativos en las formas de } \\
\text { alimentación. Conocer los } \\
\text { significados de Salud- } \\
\text { Alimentación }\end{array}$ & $\begin{array}{l}\text { Problemas de salud y } \\
\text { alimentación. Higiene } \\
\text { alimentaria. } \\
\text { Recursos didácticos }\end{array}$ \\
\hline $\begin{array}{l}\text { ¿Cómo abordar la } \\
\text { temática en el } \\
\text { mundo educativo? }\end{array}$ & $\begin{array}{l}\text { Análisis de unidades } \\
\text { didácticas. } \\
\text { Análisis de recursos } \\
\text { educativos }\end{array}$ & $\begin{array}{c}\text { Favorecer la formación } \\
\text { educativa. Analizar el interés } \\
\text { educativo de distintos recursos }\end{array}$ & $\begin{array}{l}\text { Diseño didáctico } \\
\text { Programación escolar. } \\
\text { Niveles de } \\
\text { formulación de } \\
\text { contenidos en } \\
\text { Alimentación. } \\
\text { Recursos. } \\
\end{array}$ \\
\hline
\end{tabular}

Cuadro 4: Descripción Actividades Guía, sus objetivos y contenidos a partir de situaciones problemáticas planteadas en clase. 


\section{Principales investigaciones abordadas}

Intentaremos ser sintéticos y extraer aquellas consideraciones y cuestiones más relevantes, asimismo recordemos que los datos que expondremos se pueden consultar más profundamente en: Rodrigo, 2000; Ejeda, 2006; Ejeda 2008; Rodrigo y Ejeda, 2008 y 2010; Rodrigo et al., 2009, 2010a y 2010 b.

\section{Principales problemáticas sobre las que se ha estudiado}

Las principales cuestiones de estudio se centrarían en:

- ¿Qué conocen sobre cuestiones básicas de Alimentación y qué creencias y actitudes presentan nuestros alumnos en referencia a la práctica de una dieta saludable? ¿Con la docencia mejoran los conocimientos, actitudes y creencias en torno a la Alimentación saludable?

- ¿Cuál es el estado nutricional de nuestros alumnos? ¿Cuáles son los hábitos alimenticios al comenzar la docencia? ¿Después de la acción docente han variado los hábitos dietéticos de los futuros Maestros?

\section{Diseño experimental seguido}

El diseño básico que se ha propuesto es "cuasi-experimental", una modalidad importante que es empleada dentro del ámbito de la investigación social. Este tipo de diseños ha sido muy utilizado en la investigación social y son fácilmente interpretables como señalan distintos autores (Ato, 1995; Campbell y Stanley, 1982; Gómez y Hombrados, 1988). El ejemplo prototípico sería el representado en el Cuadro 5 en el que una intervención a partir de una Asignatura "Fundamentos y didáctica de la Alimentación" (X) busca generar cambio en los "Conocimientos, actitudes y/o hábitos de los alumnos sobre Alimentación" (Y). Asimismo, previo análisis de resultados, el grupo experimental se compara con el grupo control, por lo que se controla una posible intervención de otros factores que pudieran haber contribuido a la alteración de las puntuaciones u observaciones finales.

Como se puede apreciar en el Cuadro 6 se presenta una síntesis de los principales estudios a los que nos vamos a referir a continuación.

\begin{tabular}{|c|c|c|c|}
\cline { 2 - 4 } \multicolumn{1}{c|}{ Grupos } & Pre-test & Tratamiento & Pos-test \\
\hline Experimental (GE) & & $\mathrm{X}$ & $\mathrm{Y}_{\mathrm{E} 2}$ \\
\hline Control (GC) & $\mathrm{Y}_{\mathrm{C} 1}$ & -- & $\mathrm{Y}_{\mathrm{C} 2}$ \\
\hline
\end{tabular}

Cuadro 5. Esquema del diseño investigador seguido en nuestros estudios 


\begin{tabular}{|c|c|c|c|}
\hline Publicación & Objetivo & Muestra & $\begin{array}{l}\text { Instrumento } \\
\text { empleado }\end{array}$ \\
\hline Rodrigo, 1995. & $\begin{array}{l}\text { Analizar preferencias } \\
\text { en EpS }\end{array}$ & $\begin{array}{l}318 \text { futuros } \\
\text { Maestros }\end{array}$ & $\begin{array}{l}\text { Cuestionarios Previos } \\
\text { a docencia. }\end{array}$ \\
\hline Rodrigo, 1999. & $\begin{array}{l}\text { Investigar la } \\
\text { evolución de } \\
\text { conocimientos } \\
\text { alimentarios. }\end{array}$ & 49 futuros Maestros & $\begin{array}{l}\text { Cuestionarios Pre- } \\
\text { Post. }\end{array}$ \\
\hline $\begin{array}{l}\text { Rodrigo y Ejeda, } \\
2008 .\end{array}$ & $\begin{array}{l}\text { Investigar la } \\
\text { evolución de } \\
\text { conocimientos y } \\
\text { creencias } \\
\text { alimentarias. }\end{array}$ & 147 futuros Maestros & $\begin{array}{l}\text { Cuestionarios Pre- } \\
\text { Post. }\end{array}$ \\
\hline Rodrigo y col., 2009. & $\begin{array}{l}\text { Analizar la situación } \\
\text { alimentaria }\end{array}$ & 147 futuros Maestros & $\begin{array}{l}\text { Cuestionarios Pre- } \\
\text { Post. } \\
\text { Análisis de cuadernos } \\
\text { personales. }\end{array}$ \\
\hline $\begin{array}{l}\text { Rodrigo y Ejeda, } \\
2010 .\end{array}$ & $\begin{array}{l}\text { Exponer un método } \\
\text { de análisis } \\
\text { alimentario }\end{array}$ & 50 futuros Maestros & $\begin{array}{l}\text { Análisis de cuadernos } \\
\text { personales. }\end{array}$ \\
\hline Rodrigo et al., 2010a. & $\begin{array}{l}\text { Investigar la } \\
\text { evolución de } \\
\text { conocimientos y } \\
\text { creencias } \\
\text { alimentarias. }\end{array}$ & 120 futuros Maestros & $\begin{array}{l}\text { Cuestionarios Pre- } \\
\text { Post. }\end{array}$ \\
\hline
\end{tabular}

Cuadro 6. Resumen de algunos de los principales estudios realizados.

\section{Síntesis y discusión de los resultados más relevantes}

\section{Datos de Identificación de sujetos investigados y Caracterización Nutricional}

Hasta ahora el género fundamentalmente del alumnado con el que hemos realizado tareas investigadoras ha sido el femenino (porcentaje medio del $94 \%$ ), con una edad media de 21 años. A partir del curso 2012-13 la edad media de nuestros futuros alumnos entendemos se incrementará en dos años, dado que la asignatura de referencia se dará en $4^{\circ}$ curso; asimismo la distribución de sexos puede cambiar. Esto puede tener implicaciones para investigaciones futuras dado que los niveles de energía y nutrientes recomendados se definen por edad y sexo (Moreiras et al., 2009; Requejo y Ortega, 2000)

La Caracterización Nutricional media que presenta el alumnado estudiado hasta ahora empleando para ella el Índice de Masa Corporal (IMC) y las categorías 
propuestas por la Sociedad Española para el Estudio de la Obesidad (SEEDO, 2000) de acuerdo con los sexos y las edades estudiadas sería el Normopeso para cerca del $78 \%$, seguido del Peso insuficiente para un $15 \%$, luego el Sobrepeso grado I para un $5 \%$, y por último el Sobrepeso grado II (Preobesidad) para el 2\% aproximadamente. Estos datos son similares a los que presentan estudiantes universitarios de su misma edad (Rodrigo et al., 2010b). No obstante, estos datos al ser globales, son engañosos y no aclaran las importantes desviaciones individuales de lo que deberían ser unos comportamientos alimentarios saludables más detallados que como veremos deben de mejorar.

\section{Datos sobre motivaciones y conocimientos en Alimentación}

Para la mayoría del alumnado investigado la motivación mayor para afrontar mejora de conocimientos, actitudes o hábitos relacionados con cuestiones dietéticas estaría unida a mejoras de su salud (Rodrigo et al., 2010a), en la misma línea se manifiestan futuros sanitarios en formación estudiados (Rodrigo et al., 2010b). Por lo tanto el factor salud y su mejora parece que debería estar presente en los planteamientos de cualquier diseño didáctico sobre Educación alimentaria que abordemos con estos colectivos.

La formación previa en Alimentación mayoritariamente la consideran los alumnos de tipo extraescolar y alejada de lo estudiado en su paso por los centros educativos (Rodrigo et al., 2010a).

Inicialmente constatamos en todos nuestros estudios muchas imprecisiones en conceptos básicos sobre Alimentación (Ejeda 2008, Rodrigo et al., 2010a). No obstante, se consiguen mejoras significativas conceptuales con asignaturas del tipo similar a la que proponemos, por eso reclamamos para futuros profesores, que deben ejercer en la sociedad actual, algún tipo de formación alimentaria similar a la que hemos propuesto y analizado. Además, la mejora estadísticamente significativa de conocimientos básicos sobre Alimentación es independiente de la situación de formación previa (Ciencias, Letras, etc.) con la que vienen nuestros alumnos (Ejeda, 2008).

En tal sentido, seguimos considerando clave que los futuros profesores para trabajar cuestiones sobre Alimentación deben manejar correctamente la asociación conceptual "tipo de alimento, su nutriente principal en él presente, y función dietética desempeñada por su consumo", dado que está en la base de saber elegir conscientemente un tipo de alimento u otro además de entender el significado didáctico de las Guías Alimentarias, instrumentos que consideramos imprescindibles en el saber enseñar y educar sobre Alimentación (Rodrigo y Ejeda, 2010).

Según nuestros estudios, los alumnos interpretan mucho mejor la asociación alimento con su componente nutritivo más representativo que con la función dietética que se les asigna. Así, sobre conocimientos en formación alimentaria en su momento planteamos cuatro posibles niveles de formación (Rodrigo y Ejeda, 2008) y para la propuesta didáctica con Futuros Maestros consideramos que con obtener hasta el tercero es suficiente y estos estadios serían: 
Primer nivel centrado en saber cuánto y qué hay que comer y normas básicas de higiene.... Segundo nivel, saber qué nutrientes más relevantes tiene lo que comemos.... Tercer nivel conocer qué funciones biológicas básicas (constructora-desaconsejamos el término plástico en la didáctica de la dieta-, reguladora o energética) se consiguen con los alimentos y sus nutrientes (p.245).

\section{Datos sobre Hábitos Alimentarios}

En torno a un tercio de los alumnos analizados se muestran claramente activos en buscar una elección de dieta saludable; pero para la mayoría generalmente la elección de dieta sigue la dependencia familiar (Rodrigo et al., 2010a). El periodo de estudios universitarios suele ser el momento en que los estudiantes asumen por primera vez la responsabilidad de su comida. Por tanto, se trata de un periodo de educación crítico para el desarrollo de hábitos dietéticos que tienen mucha importancia en la futura salud como ponen en evidencia distintos autores (Baric y Satalic, 2003) y estimamos que esta circunstancia hay que explotarla didácticamente.

Como instrumento didáctico de Guía de dieta o Guía Alimentaria abogamos por el trabajo con una Rueda de los Alimentos adaptada a las nuevas exigencias dietéticas (ver Ejeda, 2008; Rodrigo y Ejeda, 2010). El conocimiento de este instrumento nos resulta más útil y didáctico que otros como las Pirámides de Alimentación para el trabajo de la organización de la dieta. Con su aplicación los alumnos deben de realizar, como ya hemos expuesto, un estudio personal de sus ingestas durante una semana, muchos de los resultados que exponemos seguidamente surgen a través del análisis de sus documentos personales de estos ejercicios.

Principalmente realizan "desayuno, comida y cena" y otras dos ingestas opcionales (principalmente la merienda frente a la de media-mañana), la media está en 3,8 ingestas al día (Rodrigo et al., 2009). En estudios preliminares (Rodrigo et al., 2012) estamos observando que existen alumnos, por ejemplo, que no realizan el desayuno (del seguimiento realizado durante siete días existe un $20 \%$ aproximadamente que no desayuna alguno de esos días) y muchos no realizan la toma de media mañana (del seguimiento de siete días aproximadamente el $85 \%$ no realiza esta toma algún día) y esto consideramos debería cambiar.

La ingesta energética media diaria de la Dieta de los alumnos investigados (Rodrigo et al., 2009) es de unas $1750 \mathrm{Kcal}$. (Véase la Tabla1), dado la tipología de alumno que hemos estudiado (mujer de 21 años) se situaría esto en la banda baja de las propuestas de consumo aconsejables (Moreiras et al. 2009; Requejo y Ortega, 2000). 


\begin{tabular}{|c|c|c|c|c|c|}
\hline Macronutrientes & Kcal. & \% Kcal. & kcal / g & $\mathbf{g}$ & $\mathbf{\% g}$ \\
\hline Hidratos de Carbono & 782,3 & $45 \%$ & 3,875 & 201,9 & $57,4 \%$ \\
\hline Lípidos & 643,2 & $37 \%$ & 9 & 71,5 & $20,4 \%$ \\
\hline Proteínas & 313,0 & $18 \%$ & 4 & 78,2 & $22,2 \%$ \\
\hline Total & $\mathbf{1 . 7 3 8 , 5}$ & $100 \%$ & \multicolumn{1}{|c}{351,6} & $100 \%$ \\
\hline
\end{tabular}

Tabla 1: Ingesta media diaria de Energía y Macronutrientes $(\mathrm{n}=147$ estudiantes $)$

El Perfil Calórico de la Dieta diaria de los alumnos estudiados, con respecto a su ingesta recomendada es deficiente y desequilibrado. Consumen un exceso en kilocalorías de Proteínas y Lípidos, y un déficit de Hidratos de Carbono (Rodrigo et al., 2009). Si a esto le unimos que casi el $100 \%$ de los alumnos realizan la cena (frente a la toma de media mañana que suele ser minoritaria) y la última comida nocturna presenta una ingesta en porcentaje de kilocalorías superior a la recomendada se pueden sentar bases para desarrollos de futuros estados de pre-obesidad (véanse las Tablas 2 y 3 ).

\begin{tabular}{|c|c|c|c|c|c|}
\hline Perfil de Glúcidos & \% Alumnos & P. de Lípidos & \% Alumnos & P. de Proteínas & \% Alumnos \\
\hline$<55 \%$ & $71,3 \%$ & $<25 \%$ & $6,9 \%$ & $<10 \%$ & $0,0 \%$ \\
\hline 囚55\%-60\% & $\mathbf{2 7 , 6 \%}$ & 囚25\%-30\% & $\mathbf{1 3 , 8 \%}$ & $\circledR \mathbf{1 0} \% \mathbf{- 1 5 \%}$ & $\mathbf{4 , 6 \%}$ \\
\hline$>60 \%$ & $1,1 \%$ & $>30 \%$ & $79,3 \%$ & $>15 \%$ & $95,4 \%$ \\
\hline
\end{tabular}

®: Recomendada (véase Requejo y Ortega, 2000)

Tabla 2: Porcentajes de alumnos que se incluyen dentro de los límites de los tramos del Perfil Calórico de la Dieta para cada uno de los macronutrientes -Glúcidos, Lípidos y Proteínas- (n= 147 estudiantes)

\begin{tabular}{|c|c|c|}
\cline { 2 - 3 } \multicolumn{1}{c|}{} & Rangos Kcal & Alumnos \\
\hline Tipo de ingesta diaria & $\mathbf{\%}$ & $\mathbf{\%}$ \\
\hline \multirow{3}{*}{ Desayuno + Media Mañana } & $<20 \%$ & $24,1 \%$ \\
\cline { 2 - 3 } & $\mathbf{2 0 - 2 5 \%}{ }^{\circledR}$ & $\mathbf{2 3 , 0} \%$ \\
\cline { 2 - 3 } & $>25 \%$ & $52,9 \%$ \\
\hline \multirow{3}{*}{ Comida } & $<35 \%$ & $35,6 \%$ \\
\cline { 2 - 3 } & $\mathbf{3 5 - 4 0 \%}{ }^{\circledR}$ & $\mathbf{2 8 , 7 \%}$ \\
\cline { 2 - 3 } & $>40 \%$ & $35,6 \%$ \\
\hline \multirow{3}{*}{ Cenienda } & $<10 \%$ & $62,1 \%$ \\
\cline { 2 - 3 } & $\mathbf{1 0 - 1 5 \%}{ }^{\circledR}$ & $\mathbf{2 4 , 1 \%}$ \\
\cline { 2 - 3 } & $>15 \%$ & $13,8 \%$ \\
\hline & $<20 \%$ & $12,60 \%$ \\
\cline { 2 - 3 } & $\mathbf{2 0 - 2 5 \%}{ }^{\circledR}$ & $\mathbf{2 0 , 7 0 \%}$ \\
\cline { 2 - 3 } & $>25 \%$ & $66,70 \%$ \\
\hline
\end{tabular}

(B: Recomendada (véase Requejo y Ortega, 2000)

Tabla 3: Porcentajes comparativos entre el Perfil Calórico recomendado® y el obtenido de la

Dieta (media) para cada uno de los tipos de ingesta realizados ( $\mathrm{n}=147$ estudiantes).

La frecuencia de consumo medio por parte de los alumnos respecto de las "raciones recomendadas" (véase la Tabla 4) por grupos de alimentos según nuestra 
propuesta didáctica (Rodrigo y Ejeda, 2010) implica que sus hábitos alimenticios presentan un exceso ligero del consumo del grupo de Carnes, Pescados, Huevos, Legumbres y Frutos Secos (G2/G3a), del grupo Dulces y refrescos (G6b) y Grasas y aceites $(\mathrm{G} 7 \mathrm{a} / \mathrm{b})$; y un déficit ligero del grupo Lácteos y derivados (G1) y Cereales y Patatas (G6a/G3b), y un déficit muy acusado del grupo Verduras y Frutas (G4/G5). Por tanto, la mejora del consumo de lácteos, cereales, frutas y alimentos con mejores grasas es un objetivo de interés didáctico y está en línea con las recomendaciones dietéticas recientes para la población adulta española (ENIDE, 2011).

Por otra parte, según nuestros estudios, sólo en torno al 30\% de los alumnos realiza una dieta próxima o dentro de los límites recomendables de "dieta equilibrada y saludable" (Rodrigo et al., 2009). Parece pues de interés mejorar los porcentajes de alumnos que presenten una dieta saludable (véase la Tabla 5).

\begin{tabular}{|c|c|c|c|}
\hline \multirow[b]{2}{*}{ Grupos } & \multirow[b]{2}{*}{ Alimentos } & \multicolumn{2}{|c|}{ Ración recomendada ${ }^{\circledR}$} \\
\hline & & Semanal & Diaria \\
\hline G1 & Lácteos y derivados & $14-21 \AA$ & $2-3 \AA$ \\
\hline G1 & Leche & 7,33 & 1,14 \\
\hline G1 & Yogurt & 2,24 & 0,40 \\
\hline G1 & Queso & 2,58 & 0,32 \\
\hline G1 & Total & 12,15 & 1,86 \\
\hline
\end{tabular}

\begin{tabular}{|c|c|c|c|}
\hline G2/G3a & Carnes, Pescados, .... & $\mathbf{1 4}{ }^{\circledR}$ & $\mathbf{2}{ }^{\circledR}$ \\
\hline G2 & Carnes & 8,5 & 1,21 \\
\hline G2 & Pescados & 3,1 & 0,43 \\
\hline G2 & Huevos & 1,6 & 0,25 \\
\hline G3a & Legumbres & 1,7 & 0,21 \\
\hline G3a & Frutos Secos & 0,7 & 0,11 \\
\hline G2-3a & Total & $\mathbf{1 5 , 6}$ & $\mathbf{2 , 2 1}$ \\
\hline
\end{tabular}

\begin{tabular}{|c|c|c|c|}
\hline G4 & Verduras y Hortalizas & $7-14 \AA$ & $1-2 \AA$ \\
\hline G4 & Verduras y Hortalizas & 49 & 0.8 \\
\hline G4 & Total & 4,9 & 0,8 \\
\hline
\end{tabular}

\begin{tabular}{|c|c|c|c|}
\hline G5 & Frutas & $\mathbf{1 4 - 2 1} \AA$ & $\mathbf{2}-\mathbf{3}$ 巴 \\
\hline & & & \\
\hline G5 & Frutas & 7,4 & 1,2 \\
\hline G5 & Total & $\mathbf{7 , 4}$ & $\mathbf{1 , 2}$ \\
\hline
\end{tabular}




\begin{tabular}{|c|c|c|c|}
\hline G6-G3b & Cereales, Patatas,.. & $\mathbf{2 8}-\mathbf{3 5}{ }^{\circledR}$ & $\mathbf{4 - 5}{ }^{\circledR}$ \\
\hline \multicolumn{3}{|c|}{} \\
\hline G6a & Cereales, arroz.... & 16,9 & 2,5 \\
\hline G3b & Patatas & 1,9 & 0,3 \\
\hline G6b & Azúcar & 1,1 & 0,3 \\
\hline G6b & Dulces & 0,9 & 0,1 \\
\hline G6b & Refrescos / Zumos & 3,4 & 0,4 \\
\hline G6b & Alcohol & 0,2 & 0,0 \\
\hline G6-3b & Total & $\mathbf{2 4 , 4}$ & $\mathbf{3 , 6}$ \\
\hline
\end{tabular}

\begin{tabular}{|c|c|c|c|}
\hline G7 & Grasas y aceites & $\mathbf{7}$ ( $)$ \\
\hline \multicolumn{3}{|c|}{ ( $)$} \\
\hline G7 & Grasas y aceites & 5,21 & 0,61 \\
\hline G7 & Total & $\mathbf{5 , 2 1}$ & $\mathbf{0 , 6 1}$ \\
\hline
\end{tabular}

Nota: ${ }^{\circledR}=$ raciones recomendadas (véase Requejo y Ortega, 2000)

Tabla 4: Porcentajes de Ingesta media de cantidades físicas de ración comestible -Semanal y Diaria- $(n=50$ estudiantes $)$

\begin{tabular}{|c|c|c|c|}
\hline \multirow{2}{*}{ Necesidades } & $\begin{array}{c}\text { Grupos de } \\
\text { Alimentos }\end{array}$ & Raciones semanales ${ }^{\circledR}$ & \% Alumnos \\
\hline \multirow{2}{*}{ Constructoras } & G1 & $14-21$ & $24,1 \%$ \\
\cline { 2 - 4 } & G2/G3a & $12-15$ & $29,9 \%$ \\
\hline \multirow{2}{*}{ Reguladoras } & G4 & $7-14$ & $23,0 \%$ \\
\cline { 2 - 4 } & $\mathbf{G 5}$ & $14-21$ & $18,4 \%$ \\
\hline \multirow{2}{*}{ Energéticas } & G6/G3b & $28-35$ & $34,5 \%$ \\
\cline { 2 - 4 } & $\mathbf{G 7}$ & $7-10$ & $17,2 \%$ \\
\hline
\end{tabular}

Nota: ${ }^{\circledR}=$ raciones recomendadas (véase Requejo y Ortega, 2000)

Tabla 5: Porcentajes de alumnos que cubren las necesidades energéticas, constructoras (o plásticas) y reguladoras recomendadas $®$ para cada grupo de alimentos ( $\mathrm{n}=50$ estudiantes).

Datos sobre creencias en valoración global de dieta.

Los alumnos que toman conciencia crítica de sus hábitos alimenticios (sean buenos o malos) antes del curso de Alimentación están en torno al 60\%; después de la acción didáctica dicho porcentaje tiende a mejorar (cerca de un 75\% valora adecuadamente su dieta), y es estadísticamente significativo (Ejeda, 2008; Rodrigo et al., 2010a). No obstante, los alumnos que parecen más formados y con mejores condiciones de dieta suelen ser más realistas y los demás parecen más permisivos y optimistas en la valoración de su dieta (véase en este sentido también un estudio con futuros sanitarios realizado en Rodrigo et al., 2010b). 


\section{Conclusiones}

En resumen, los conocimientos iniciales sobre Alimentación son muy deficientes y mejoran claramente con el desarrollo de la asignatura expuesta, por eso reclamamos para futuros Maestros tanto de Infantil como de Primaria, que deben ejercer en la sociedad actual, algún tipo de formación alimentaria similar a la que hemos propuesto y analizado. Además, la mejora estadísticamente significativa de conocimientos básicos sobre Alimentación es independiente de la situación de formación previa (Ciencias, Letras, etc.) con la que vienen nuestros alumnos.

Proponemos una secuencia de niveles conceptuales sobre Educación Alimentaria que empezarían por aspectos simples de conocimientos de consumo de alimentos y normas básicas de higiene y culminarían al final con la integración mental y conceptual del trinomio: Alimento-tipo de nutrientes relevantes en el presentes-funciones dietéticas desempeñadas.

En el segundo plano estudiado, el de la aproximación a la situación alimentaria, hemos observado que la caracterización nutricional media mayoritaria que presenta el alumnado estudiado hasta ahora, empleando para ella el Índice de Masa Corporal (IMC), es el Normopeso. No obstante la ingesta energética media diaria de la Dieta de los alumnos investigados es de unas 1750 Kcal., estando en el límite de lo recomendable para su edad. De hecho sólo en torno al 30\% de los alumnos estudiados realiza una dieta próxima o dentro de los límites recomendables de "dieta equilibrada y saludable". Parece pues de interés mejorar los porcentajes de alumnos que presenten una dieta saludable.

El campo de la mejora de hábitos alimentarios es el más difícil de educar y trasformar siendo los resultados obtenidos hasta ahora mejorables aunque positivos. En esta línea nos afirmamos en el principio de que manejar la competencia de "saber analizar críticamente el tipo de dieta semanal que se realiza" sigue siendo un objetivo clave para poder a la larga mejorar los comportamientos dietéticos de nuestros alumnos y estará presente en nuestro diseño didáctico e investigador futuro. Dentro de esta línea didáctica nos parece nuclear que los alumnos entiendan y manejen las Guías Alimentarias dado que las consideramos esenciales en la Educación Alimentaria tanto de ellos como en su preparación para posibles futuras labores docentes.

De todo lo anterior surge nuestra apuesta didáctica para el futuro próximo de intentar mejorar la dieta de nuestro colectivo de estudiantes utilizando el desayuno y potenciando la toma de media mañana como equilibradores globales de dieta. Así buscaremos mejorar el consumo de lácteos, cereales y frutas; disminuir los consumos de grasas saturadas y azucares refinados (presentes en bollería industrial, falsos zumos, golosinas /snacks de picoteo y refrescos entre horas). Entendemos que ello mejoraría el desequilibrio del perfil de dieta e incrementaría algo la ingesta energética media diaria que hemos visto parece algo escasa, esta apuesta está en consonancia con recomendaciones dietéticas recientes para la población española (ENIDE, 2011; Galiano Segovia y Moreno, 2010). 
Para finalizar, queremos manifestar nuestro agradecimiento al alumnado que ha cursado la asignatura de "La Alimentación humana en la escuela" desde el curso 199798 y ha participado voluntariamente en estas investigaciones, así reconocemos que con su interés e ilusión nos han seguido motivando para trabajar e investigar sobre algo tan actual y con tanto interés social como la mejora de la Educación en Alimentación tanto de ellos como en su día de sus futuros alumnos.

\section{Referencias bibliográficas}

ATO, M. (1995). Tipología de los diseños cuasi-experimentales, en M. T. Anguera, J. Arnau, M. Ato, R. Martínez, J. Pascual y G. Vallejo (eds.), Métodos de investigación en Psicología. Madrid: Síntesis.

BARIC, I y SATALIC, Z. (2003). Nutritive value of meals, dietary habits and nutritive status in Croatian university students according to gender. International Journal Food Science Nutrition, 54 (6): 473-484.

BOE (2011). Ley 17/2011 (BOE no 160 de 6 de julio) de Seguridad Alimentaria y de Nutrición.

BOE (2006a). REAL DECRETO 1513/2006 por el que se establecen las enseñanzas mínimas de la Educación Primaria.

BOE (2006b). REAL DECRETO 1630/2006, de 29 de diciembre, por el que se establecen las enseñanzas mínimas del segundo ciclo de Educación Infantil.

BOE (2006c). REAL DECRETO 1631/2006 por el que se establecen las enseñanzas mínimas correspondientes a la Educación Secundaria Obligatoria.

CAMPBELL, D. T. y STANLEY, J.C. (1982). Diseños experimentales y cuasiexperimentales en la investigación social. Buenos Aires: Amorrortu.

ENIDE (2011). Encuesta Nacional de Ingesta Dietética Española. Recuperado de http://www.aesan.msps.es/ (Consultado el 4 de julio de 2012).

EJEDA, J. M. (2006). El conocimiento sobre Alimentación en la Formación Inicial de Maestros. Trabajo de investigación de final de Programa de Doctorado (inédito). Madrid: Facultad de Educación- UCM.

EJEDA, J. M. (2008). El conocimiento sobre Alimentación en la Formación Inicial de Maestros. Tesis Doctoral (Dirigida por M. Rodrigo). Madrid: UCM.

GALIANO SEGOVIA, M. J. y MORENO, J. M. (2010). El desayuno en la infancia: más que una buena costumbre. Acta Pediátrica. Española, 68(8), 403-408.

GAVIDIA, V., RODES, J. M. y CARRATALÁ, A. (1993). La Educación para la Salud. Una propuesta fundamentada desde el campo de la docencia. Enseñanza de las Ciencias, 11(3), 289-296. 
GÓMEZ, L. y HOMBRADOS, I. (1988). Diseños de intervención comunitaria, en A. Martín, F. Chacón y M. Martínez (eds.), Psicología Comunitaria, 149-166. Madrid: Visor.

LÓPEZ NOMDEDEU, C. (1974). Alimentación práctica para el hogar. Programa en Educación en Alimentación y Nutrición (EDALNU). Madrid: Dirección General de Sanidad.

MARTÍNEZ, J. R., PALENCIA, A., SERRANO, L. y VILLARINO, A. (2007). Educación alimentaria escolar y extraescolar. Programas y didáctica, en J. R. Martínez e I. Polanco (coord.), El libro blanco de la Alimentación escolar, 137156. Madrid: Interamericana.

MOREIRAS, O., CARVAJAL, A., CABRERA, L. Y CUADRADO, C. (2009). Tabla de composición de Alimentos. Madrid: Pirámide.

OMS (1998). Organización Mundial de la Salud. Promoción de la Salud. Glosario. Ginebra: Ediciones de la OMS.

OMS (2002). 55 Asamblea sobre Régimen Alimentario, Actividad fisica y Salud. Ginebra: Ediciones de la OMS.

OMS (2006). Marco para el seguimiento y evaluación de la aplicación de la Estrategia Mundial sobre Régimen Alimentario, Actividad Física y Salud (DPAS). Ginebra: Ediciones de la OMS.

REQUEJO, A. M. Y ORTEGA, R. M. (2000). Nutriguía. UCM: Madrid.

RODRIGO VEGA, M. (1995). Concepciones de los futuros profesores de Primaria sobre Educación para la Salud. Revista Interuniversitaria de Formación del Profesorado, 24: 173-180.

RODRIGO, M. (1999). Puesta en práctica de una asignatura de alimentación en Formación Inicial del profesorado. Revista Complutense de Educación, 10 (1): 379-391.

RODRIGO, M. (2000). Una Asignatura de Alimentación en Formación Inicial del Profesorado. Reflexiones sobre la Didáctica de las Ciencias Experimentales. Actas de los XIX Encuentros de Didáctica de las Ciencias Experimentales. Madrid: Universidad Complutense de Madrid, pp. 268-274.

RODRIGO, M. Y EJEDA, J. M. (2008). Concepciones sobre Alimentación en futuros profesores. Construcción del Conocimiento Pedagógico. Teoría de la Educación, 20,225-247.

RODRIGO, M., EJEDA, J. M. Y SÁNCHEZ, S. (2009). La enseñanza de la Alimentación en futuros Maestros: estudio de estado nutricional y hábitos alimentarios. Enseñanza de las Ciencias, $n^{\circ}$ extra VIII Congreso Internacional sobre Investigación en Didáctica de las Ciencias, 804-811. 
RODRIGO, M. Y EJEDA, J. M. (2010). La Rueda de los Alimentos. Propuesta para la formación de Maestros. Alambique, 65, 52-60.

RODRIGO, M., EJEDA, J. M. Y GONZÁLEZ, C. (2010a). Una investigación en torno a las concepciones sobre Alimentación en futuros profesores. Revista Complutense de Educación, 21(1), 189-207.

RODRIGO, M., EJEDA, J. M. Y MANJARREZ, T. (2010b). Análisis de los conocimientos en Alimentación de futuros sanitarios. Implicaciones pedagógicas. Teoría de la Educación. Revista Interuniversitaria, 22(1), 163-195.

RODRIGO, M., IGLESIAS, M ${ }^{\mathrm{a}}$ T., EJEDA, J. M., GONZÁLEZ, M ${ }^{\mathrm{a}}$ P. Y MIJANCOS, $M^{a}$ T. (2012). Educación Alimentaria con futuros Maestros: Hábitos de desayuno. Nutrición Clín. Diet. Hospitalaria, 32 (Supl.: Actas de las XVI Jornadas de Nutrición Práctica), 30.

SEEDO (2000). Sociedad Española para el Estudio de la Obesidad. Consenso para la evaluación del Sobrepeso y la Obesidad y el establecimiento de criterios de intervención terapéutica. Medicina Clínica, 115, 587-597.

WHO (1986). Ottawa Charter for Health Promotion. First International Conference on Health Promotion. Ottawa, Canadá, 17-21 November.

\section{Correspondencia con los autores:}

Maximiliano RODRIGO VEGA

Departamento de Didáctica de las Ciencias Experimentales

Facultad de Educación

C/Rector Royo Villanova s/n. 28040 Madrid

e mail: mrodrig1@edu.ucm.es

Teléfono: 91-3946280. 